\title{
Study on initial geometry fluctuations via participant plane correlations in heavy ion collisions: part II
}

\author{
Jiangyong Jia ${ }^{1,2, a}$, Derek Teaney ${ }^{3}$ \\ ${ }^{1}$ Chemistry Department, Stony Brook University, Stony Brook, NY 11794, USA \\ ${ }^{2}$ Physics Department, Brookhaven National Laboratory, Upton, NY 11796, USA \\ ${ }^{3}$ Department of Physics and Astronomy, Stony Brook University, Stony Brook, NY 11794-3800, USA
}

Received: 25 June 2013 / Revised: 16 August 2013 / Published online: 2 October 2013

(C) The Author(s) 2013. This article is published with open access at Springerlink.com

\begin{abstract}
Further investigation of the participant plane correlations within a Glauber model framework is presented, focusing on correlations between three or four participant planes of different order. A strong correlation is observed for $\cos \left(2 \Phi_{2}^{*}+3 \Phi_{3}^{*}-5 \Phi_{5}^{*}\right)$ which is a reflection of the elliptic shape of the overlap region. The correlation between the corresponding experimental event plane angles can be easily measured. Strong correlations of similar geometric origin are also observed for $\cos \left(2 \Phi_{2}^{*}+4 \Phi_{4}^{*}-6 \Phi_{6}^{*}\right), \cos \left(2 \Phi_{2}^{*}-\right.$ $\left.3 \Phi_{3}^{*}-4 \Phi_{4}^{*}+5 \Phi_{5}^{*}\right), \cos \left(6 \Phi_{2}^{*}+3 \Phi_{3}^{*}-4 \Phi_{4}^{*}-5 \Phi_{5}^{*}\right)$, $\cos \left(\Phi_{1}^{*}-2 \Phi_{2}^{*}-3 \Phi_{3}^{*}+4 \Phi_{4}^{*}\right), \cos \left(\Phi_{1}^{*}+6 \Phi_{2}^{*}-3 \Phi_{3}^{*}-4 \Phi_{4}^{*}\right)$, and $\cos \left(\Phi_{1}^{*}+2 \Phi_{2}^{*}+3 \Phi_{3}^{*}-6 \Phi_{6}^{*}\right)$, which are also measurable. Experimental measurements of the corresponding event plane correlators in heavy ion collisions at RHIC and the LHC may improve our understanding of the physics underlying the measured higher order flow harmonics.
\end{abstract}

In a previous paper [1], one of us proposed a method for measuring the correlations between several event planes of different order. We estimated the magnitude of these correlations in configuration space via a Monte Carlo Glauber model, and several strong spatial correlators were identified. Despite the possible non-linear mixing between harmonics of different order in the hydrodynamic evolution [2-4], these geometric correlations may still survive and contribute to the event plane correlations in momentum space. For a detailed introduction and motivation on this subject, we refer to Ref. [1].

In this paper we discuss several geometric correlators involving three and four participant planes, which are of current experimental interest. These correlators are not covered

a e-mail: jjia@bnl.gov in the literature, although some related correlators have been studied both numerically [5-7] and analytically [9].

As pointed out in Ref. $[1,7,8]$, the event plane correlations that can be measured experimentally involve various linear combinations of the $n$-th order planes $\Phi_{n}, c_{1} \Phi_{1}+$ $2 c_{2} \Phi_{2} \ldots+l c_{l} \Phi_{l}$, where the integers $\left(c_{1} \ldots c_{l}\right)$ satisfy the constraint

$c_{1}+2 c_{2} \cdots+l c_{l}=0$.

Due to this constraint, only $l-1$ angles are independent. The differential distribution in this observable is an even function and can be expanded into a Fourier series

$$
\begin{aligned}
\frac{d N_{\mathrm{evts}}}{d\left(c_{1} \Phi_{1}+\cdots+l c_{l} \Phi_{l}\right)} \propto & +2 \sum_{j=1}^{\infty} V_{c_{1} \Phi_{1}, \ldots, l c_{l} \Phi_{l}}^{j} \\
& \times \cos j\left(c_{1} \Phi_{1}+\cdots+l c_{l} \Phi_{l}\right),
\end{aligned}
$$

where

$V_{c_{1} \Phi_{1}, \ldots, l c_{l} \Phi_{l}}^{j}=\left\langle\cos j\left(c_{1} \Phi_{1}+\cdots+l c_{l} \Phi_{l}\right)\right\rangle$.

The Fourier coefficients can be determined from the experimentally measured event plane angle $\Psi_{n}$ and associated resolution factor $\operatorname{Res}\left\{j c_{n} n \Psi_{n}\right\}$

$V_{c_{1} \Phi_{1}, \ldots, l c_{l} \Phi_{l}}^{j}=\frac{\left\langle\cos j\left(c_{1} \Psi_{1}+\cdots+l c_{l} \Psi_{l}\right)\right\rangle}{\operatorname{Res}\left\{j c_{1} \Psi_{1}\right\} \cdots \operatorname{Res}\left\{j c_{l} l \Psi_{l}\right\}}$,

where

$\operatorname{Res}\left\{j c_{n} n \Psi_{n}\right\}=\left\langle\cos j c_{n} n\left(\Psi_{n}-\Phi_{n}\right)\right\rangle$.

The precision with which these event plane correlations can be measured is limited by the magnitude of the resolution, which is expressed in terms of the resolution parameter $\chi_{n}[10]$ : 
$\operatorname{Res}\left\{m n \Psi_{n}\right\}$

$$
\begin{aligned}
& =\frac{\chi_{n} \sqrt{\pi}}{2} e^{-\frac{\chi_{n}^{2}}{2}}\left[I_{(m-1) / 2}\left(\frac{\chi_{n}^{2}}{2}\right)+I_{(m+1) / 2}\left(\frac{\chi_{n}^{2}}{2}\right)\right] \\
& \approx \begin{cases}1-\frac{m^{2}}{8 z}+\frac{m^{2}\left(m^{2}-4\right)}{128 z^{2}} & \text { for large } \chi_{n}, \\
-\frac{m^{2}\left(m^{2}-4\right)\left(m^{2}-16\right)}{3072 z^{3}}, z=\chi_{n}^{2} / 2 & \text { for small } \chi_{n} . \\
\frac{\sqrt{\pi}}{2^{m} \Gamma\left(\frac{m+1}{2}\right)} \chi_{n}^{m} & \end{cases}
\end{aligned}
$$

In general, $\chi_{n}$ and hence $\operatorname{Res}\left\{m n \Psi_{n}\right\}$ decrease quickly for increasing $n$. For event plane measured in $3<|\eta|<5$ in $\mathrm{Pb}+\mathrm{Pb}$ collisions at the LHC, the ATLAS Collaboration shows that $\chi_{n}$ decreases from about 2 for $n=2$ to about 0.08 for $n=6$, and is negligible for $n \geq 7$ [11]. In contrast, $\operatorname{Res}\left\{m n \Psi_{n}\right\}$ decreases more slowly with $m$ at fixed $n$, especially for $n=2$ and 3 cases where $\chi_{n} \gtrsim 1$. The dependence of $\operatorname{Res}\left\{m n \Psi_{n}\right\}$ on $n$ and $m$ limits the types of correlations that are accessible to the experiments.

The three-plane correlator can be generally expressed as a linear combination of two two-plane correlators

$$
\begin{aligned}
c_{n} n \Phi_{n}+c_{m} m \Phi_{m}+c_{l} l \Phi_{l}= & c_{m} m\left(\Phi_{m}-\Phi_{n}\right) \\
& +c_{l} l\left(\Phi_{l}-\Phi_{n}\right)
\end{aligned}
$$

which is redefined in terms of $\Phi_{a, b} \equiv\left(\Phi_{a}-\Phi_{b}\right)$

$c_{n} n \Phi_{n}+c_{m} m \Phi_{m}+c_{l} l \Phi_{l}=c_{m} \Phi_{m, n}+c_{l} \Phi_{l, n}$.

Here $n<m<l$, and we have used the constraint in Eq. (1). We shall refer to these three plane correlations as "l-m-n" correlations.

The correlation signals can be accessed via a Fourier expansion of the event distribution in ${ }^{1}\left(\Phi_{m, n}, \Phi_{l, n}\right)$ :

$$
\begin{aligned}
\frac{d^{2} N_{\mathrm{evts}}}{d \Phi_{m, n} d \Phi_{l, n}} \propto & +2 \sum_{j=1}^{\infty} V_{m, n}^{j} \cos j \Phi_{m, n}+V_{l, n}^{j} \cos j \Phi_{l, n} \\
& +2 \sum_{i, j=1}^{\infty} V_{l, m, n}^{i, \pm j} \cos \left(i \Phi_{m, n} \pm j \Phi_{l, n}\right)
\end{aligned}
$$

The meaningful coefficients are those that satisfy the constraint of Eq. (1): $(j m \bmod n)=0$ for $V_{m, n}^{j},(j l \bmod n)=0$ for $V_{l, n}^{j}$, and $(i m \pm j l \bmod n)=0$ for $V_{l, m, n}^{i, \pm j}$.

The discussion so far involves experimentally measured correlations between the true event plane angles $\Phi_{n}$ or measured event plane angles $\Psi_{n}$, which are defined in the momentum space. These correlations are partially related to

\footnotetext{
${ }^{1}$ This expression can be obtained from a double Fourier series involving $\cos i \Phi_{m, n} \cos j \Phi_{l, n}$ and $\sin i \Phi_{m, n} \sin j \Phi_{l, n}$ and, in principle, the corresponding mixed terms. However, terms linear in sine vanish since the event distribution is even under, $\Phi_{m, n} \rightarrow-\Phi_{m, n}$.
}

analogous correlations between participant planes $\Phi_{n}^{*}$ in the initial geometry. Previous study of participant plane correlations focused on three-plane correlators containing $\Phi_{1}^{*}$. In this work, we explore three-plane correlators that do not involve $\Phi_{1}^{*}$, as well as various four-plane correlators. These correlations are estimated with Monte Carlo Glauber simulations of $\mathrm{Au}+\mathrm{Au}$ collisions using a nucleon-nucleon crosssection of $\sigma=42 \mathrm{mb}$ [12]. The $\Phi_{n}^{*}$ and the eccentricity, $\epsilon_{n}$, are defined through the distribution of participants and binary collisions in the transverse plane, with a weight of $\delta=0.14$ for binary collisions, and $(1-\delta) / 2=0.43$ for participants [14]

$\epsilon_{n} e^{i n \Phi_{n}^{*}} \equiv \frac{\left\langle r^{2} e^{i n \phi}\right\rangle}{\left\langle r^{2}\right\rangle}$.

Here $(r, \phi)$ are measured relative to the weighted center of mass [13]. Alternatively, $\Phi_{n}^{*}$ can be defined with an $r^{3}$ weight for $n=1$, and a $r^{n}$-weight for $n>1$, and this definition is referred to as $r^{n}$-weighting. We also calculated the participant plane angles with CGC simulations using both the $r^{2}$ and the $r^{n}$-weighting [15, 16]. Finally, we note that $\Phi_{n}^{*}$ defines the major axes of the eccentricity [1] and is rotated by $\pi / n$ relative to traditional definition based on the minor axis.

Two interesting three-plane correlators are,

$c_{2} \Phi_{2}^{*}+3 c_{3} \Phi_{3}^{*}+5 c_{5} \Phi_{5}^{*}=c_{3} \Phi_{3,2}^{*}+c_{5} \Phi_{5,2}^{*}$,

and

$c_{2} \Phi_{2}^{*}+4 c_{4} \Phi_{4}^{*}+6 c_{6} \Phi_{6}^{*}=c_{4} \Phi_{4,2}^{*}+c_{6} \Phi_{6,2}^{*}$.

Figure 1 summarizes the "5-3-2" correlations present in Glauber and CGC simulations for the two different weighting schemes. A strong diagonal correlation is observed, corresponding to $(i, j)=(i,-i)$ or $\left\langle\cos i\left(2 \Phi_{2}^{*}+3 \Phi_{3}^{*}-5 \Phi_{5}^{*}\right)\right\rangle$. The coefficients are nearly zero for other values of $(i, j)$. The origin of this correlation is similar to the well known "3-2-1" correlation, $\left\langle\cos \left(\Phi_{1}^{*}+2 \Phi_{2}^{*}-3 \Phi_{3}^{*}\right)\right\rangle[6]$.

Specifically, both of these correlations are geometric and of order $\epsilon_{2}$. To see this we will use a simplified (but less accurate) version of the independent cluster model [9] and compute a closely related correlation function

$\frac{\left\langle\epsilon_{3} \epsilon_{5} \cos \left(2 \Phi_{2}^{*}+3 \Phi_{3}^{*}-5 \Phi_{5}^{*}\right)\right\rangle}{\sqrt{\left\langle\epsilon_{3}^{2}\right\rangle\left\langle\epsilon_{5}^{2}\right\rangle}}$.

In the cluster model, $N$ independent clusters are drawn from a distribution $\bar{n}(\boldsymbol{x})$, which is the average number of clusters per unit area in the transverse plane, with $\boldsymbol{x}=(x, y)$. $\bar{n}(\boldsymbol{x})$ is proportional to the participant density in an optical Glauber model. The cluster density in a specific event 

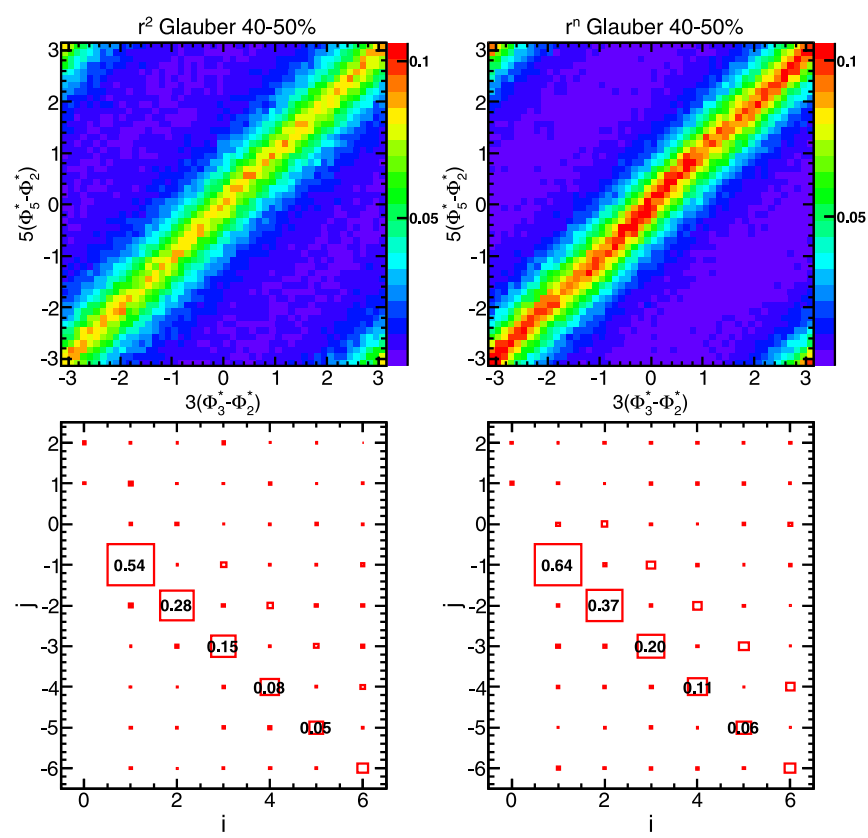

Fig. 1 The normalized event distribution for $3\left(\Phi_{3}^{*}-\Phi_{2}^{*}\right)$ and $5\left(\Phi_{5}^{*}-\Phi_{2}^{*}\right)$, i.e. $\mathrm{d}^{2} N_{\text {evts }} /\left[\mathrm{d} \Phi_{3,2}^{*} \mathrm{~d} \Phi_{5,3}^{*}\right]$ (top row). The corresponding Fourier coefficients (bottom row) in 40-50 \% centrality class, see

is $n(\boldsymbol{x})=\delta n(\boldsymbol{x})+\bar{n}(\boldsymbol{x})$ where $\delta n(\boldsymbol{x})$ satisfies Poisson statistics

$\langle\delta n(\boldsymbol{x}) \delta n(\boldsymbol{y})\rangle=\bar{n}(\boldsymbol{x}) \delta^{2}(\boldsymbol{x}-\boldsymbol{y})$.

For a given event, the odd participant planes and their angles are given by the integral

$\epsilon_{n} e^{i n\left(\Phi_{n}^{*}-\Phi_{R}^{*}\right)} \simeq \frac{1}{N\left\langle r^{2}\right\rangle} \int \mathrm{d}^{2} \boldsymbol{x} \delta n(\boldsymbol{x}) r_{x}^{2} e^{i n\left(\phi_{x}-\Phi_{R}^{*}\right)}$,

where the $\phi_{x}$ is the azimuthal angle of the cluster, and we are measuring all angles with respect to the original event plane $\Phi_{R}^{*} \simeq \Phi_{2}^{*}+\pi / 2$, and are working to leading order in $1 / N$. Multiplying Eq. (14) by its conjugate and averaging over the statistics of $\delta n(\boldsymbol{x})$, we find

$\left\langle\epsilon_{n}^{2}\right\rangle \simeq \frac{\left\langle r^{4}\right\rangle}{N\left\langle r^{2}\right\rangle^{2}}$,

which explains (again) why $\epsilon_{n}$ does not decrease with $n$ in the Glauber model [9]. Similarly, after constructing $\epsilon_{3} \epsilon_{5} e^{i\left(2 \Phi_{R}^{*}+3 \Phi_{3}^{*}-5 \Phi_{5}^{*}\right)}$ with Eq. (14), and averaging over $\delta n(\boldsymbol{x})$, we find

$\frac{\left\langle\epsilon_{5} \epsilon_{3} \cos \left(2 \Phi_{2}^{*}+3 \Phi_{3}^{*}-5 \Phi_{5}^{*}\right)\right\rangle}{\sqrt{\left\langle\epsilon_{3}^{2}\right\rangle\left\langle\epsilon_{5}^{2}\right\rangle}} \simeq-\frac{\left\langle r^{4} \cos \left(2\left(\phi_{x}-\Phi_{R}^{*}\right)\right)\right\rangle}{\left\langle r^{4}\right\rangle}$.

Equation (16) clearly shows the geometric origin of these correlations. In fact, all " $(n+2)-n-2$ ” correlations with $n$
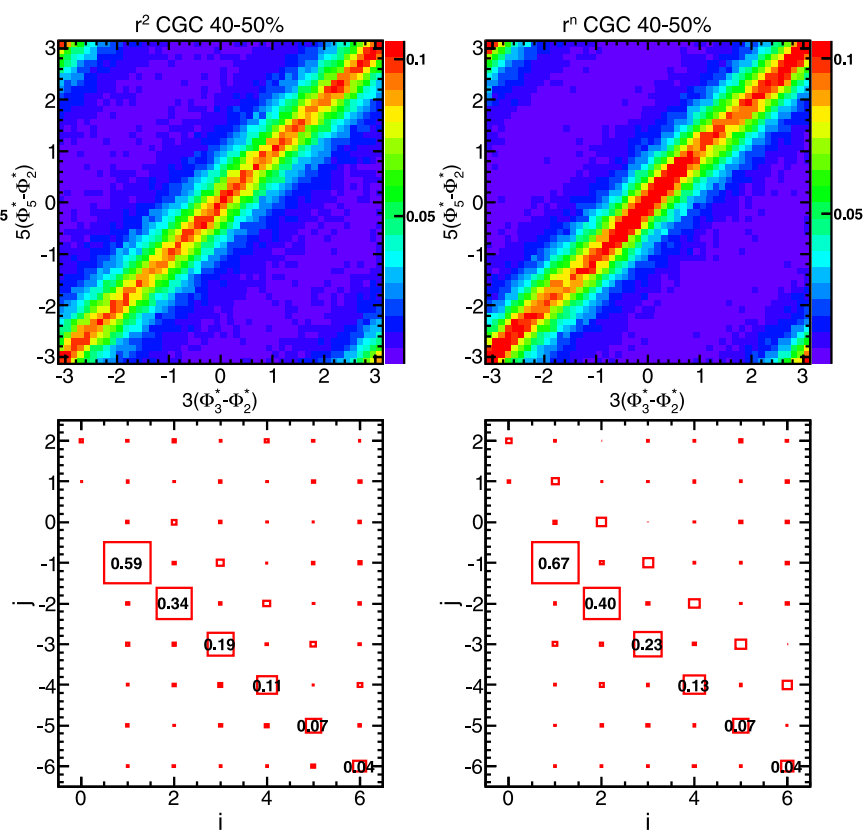

Eq. (8). Note that the constraint in Eq. (1) requires $i, j$ to be both even or both odd (Color figure online)

odd are equal, and given by Eq. (16). When the independent source model is improved by including the shift in the center of mass and terms suppressed by $1 / N$, it quantitatively describes the results of full Monte-Carlo Glauber and CGC simulations [9].

A similar correlation in the Glauber model is studied in Fig. 2 which examines the "6-4-2" three plane correlators. The largest term corresponding to $\left\langle\cos \left(2 \Phi_{2}^{*}+4 \Phi_{4}^{*}-6 \Phi_{6}^{*}\right)\right\rangle$, is much bigger than $\left\langle\cos 6\left(\Phi_{6}^{*}-\Phi_{2}^{*}\right)\right\rangle$ and almost as big as $\left\langle\cos 4\left(\Phi_{4}^{*}-\Phi_{2}^{*}\right)\right\rangle$. This term captures the strong positive correlation of $\Phi_{6}^{*}$ and $\Phi_{4}^{*}$ relative to the $\Phi_{2}^{*}$ plane as shown in the top panels of Fig. 2. However, in contrast to the "5-3-2" correlation in Fig. 1, the alignment of $\Phi_{6}^{*}, \Phi_{4}^{*}$ relative to $\Phi_{2}^{*}$ is not strictly along the diagonal $\left(\Phi_{6,2}^{*} \approx\right.$ $\left.1.7 \Phi_{4,2}^{*}\right)$, leading to large coefficients along $(i, j)=(i,-i)$, $(i,-i+1)$ and $(i,-i+2)$. This non-diagonal behavior reflects a strong influence of average geometry to even-order participant planes $\Phi_{4}^{*}, \Phi_{6}^{*}, \ldots$, which leads to strong correlations between $\Phi_{2 n}^{*}$ and $\Phi_{2}^{*}$, and hence narrow peak in distributions of $\Phi_{4,2}^{*}$ and $\Phi_{6,2}^{*}$ [1]. After removing these twoplane correlation components

$\frac{\mathrm{d}^{2} N_{\text {evts }}}{\mathrm{d} \Phi_{4,2}^{*} \mathrm{~d} \Phi_{6,2}^{*}}-\frac{\mathrm{d} N_{\text {evts }}}{\mathrm{d} \Phi_{4,2}^{*}} \frac{\mathrm{d} N_{\text {evts }}}{\mathrm{d} \Phi_{6,2}^{*}}$,

the resulting 2-D distribution is modulated around diagonal direction and with a similar magnitude as in the "5-32 " case. This harmonic variation around the diagonal band (also see Fig. 1(b) and (d)) can be analyzed similar to the "1-2-3" case [6]. 

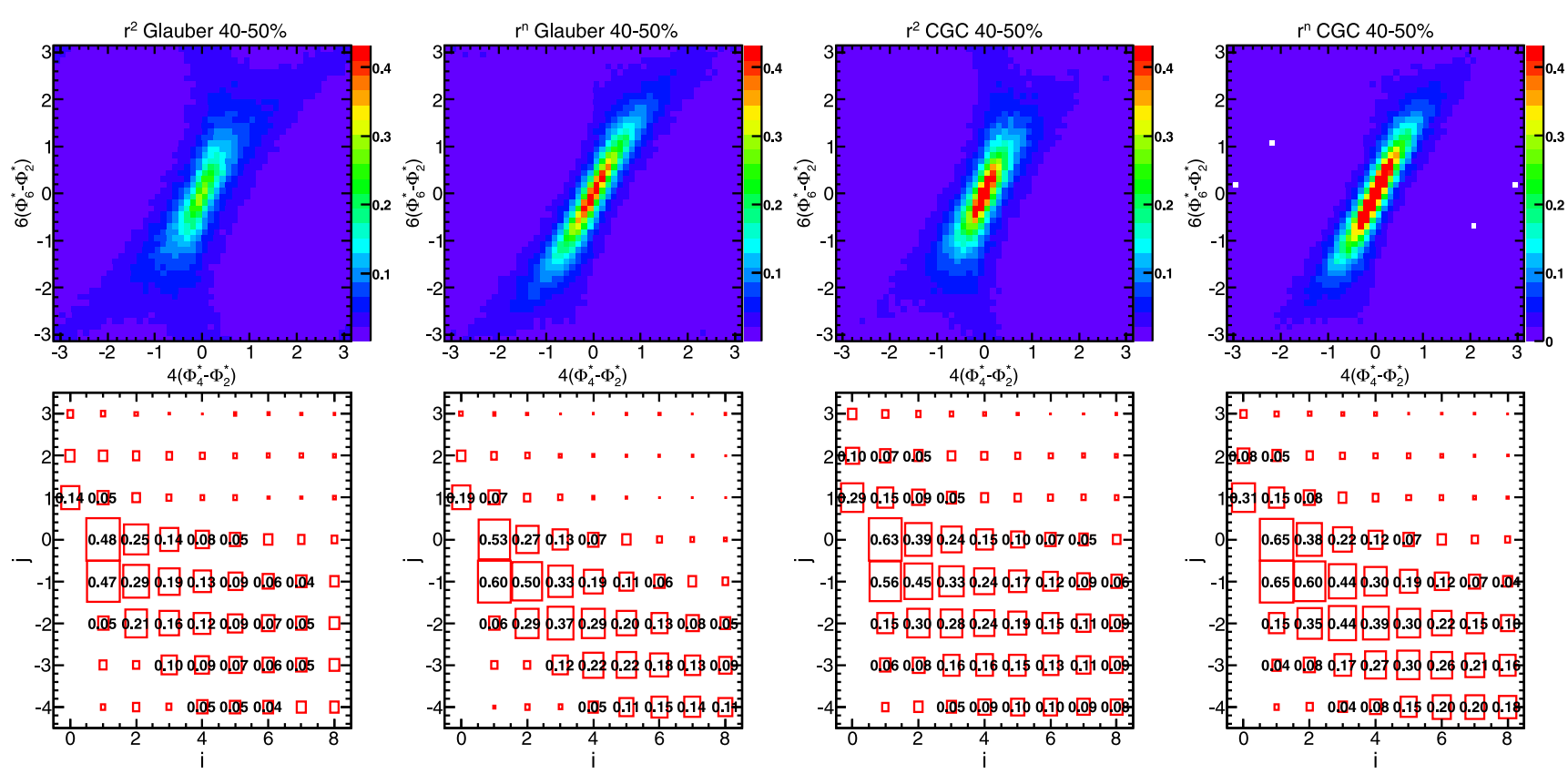

Fig. 2 The normalized event distribution for $4\left(\Phi_{4}^{*}-\Phi_{2}^{*}\right)$ and $6\left(\Phi_{6}^{*}-\Phi_{2}^{*}\right)$, i.e. $\mathrm{d}^{2} N_{\text {evts }} /\left[\mathrm{d} \Phi_{4,2}^{*} \mathrm{~d} \Phi_{6,2}^{*}\right]$ (top row). The corresponding Fourier coefficients (bottom row) in 40-50\% centrality class, see Eq. (8) (Color figure online)

Figure 3 shows the centrality dependence of several three-plane correlators for which the corresponding experimental event planes may still have decent resolution. Very strong signals are observed for $\left\langle\cos \left(2 \Phi_{2}^{*}+3 \Phi_{3}^{*}-5 \Phi_{5}^{*}\right)\right\rangle$ and $\left\langle\cos \left(2 \Phi_{2}^{*}+4 \Phi_{4}^{*}-6 \Phi_{6}^{*}\right)\right\rangle$; the signals are even bigger than $\left\langle\cos \left(\Phi_{1}^{*}+2 \Phi_{2}^{*}-3 \Phi_{3}^{*}\right)\right\rangle$ and are comparable to $\left\langle\cos 4\left(\Phi_{2}^{*}-\Phi_{4}^{*}\right)\right\rangle$. The value of $\left\langle\cos \left(2 \Phi_{2}^{*}-8 \Phi_{4}^{*}+6 \Phi_{6}^{*}\right)\right\rangle$ is also large. In contrast, the values for other correlators are small or even slightly negative for the $r^{2}$-weighting in midcentral collisions.

The behavior of these correlators towards central collisions is also quite interesting. This is the region where all the correlations are presumably dominated by fluctuations of participating nucleons. The values of $\left\langle\cos \left(2 \Phi_{2}^{*}+\right.\right.$ $\left.\left.3 \Phi_{3}^{*}-5 \Phi_{5}^{*}\right)\right\rangle$ and $\left\langle\cos \left(2 \Phi_{2}^{*}+4 \Phi_{4}^{*}-6 \Phi_{6}^{*}\right)\right\rangle$ seem to reach a constant at $10 \%$ for $N_{\text {part }}>300$, at least for the Glauber model. Similar observations are also made previously [1] for $\left\langle\cos 4\left(\Phi_{2}^{*}-\Phi_{4}^{*}\right)\right\rangle,\left\langle\cos 6\left(\Phi_{3}^{*}-\Phi_{6}^{*}\right)\right\rangle,\left\langle\cos 2\left(\Phi_{1}^{*}-\Phi_{2}^{*}\right)\right\rangle$, $\left\langle\cos \left(\Phi_{1}^{*}+2 \Phi_{2}^{*}-3 \Phi_{3}^{*}\right)\right\rangle$ and $\left\langle\cos \left(\Phi_{1}^{*}+3 \Phi_{3}^{*}-4 \Phi_{4}^{*}\right)\right\rangle$. Since the non-linear mixing between different harmonics due to hydrodynamic evolution is expected to be relatively small in central collisions, measuring the corresponding event plane correlators in this region may provide some handle on the relative role of the linear and non-linear response [3, 4].

The four-plane correlator in the Glauber and CGC models can be analyzed with a Fourier analysis similar to Eq. (8). Instead of presenting a general analysis, we will simply discuss several participant plane correlators that have a large signal and acceptable reaction plane resolution for the corresponding momentum space measurements.
Figure 4 shows the several four-plane correlators which do not involve the $\Phi_{1}^{*}$ plane. The strong signal observed for four of the correlators shown can be understood as the intercorrelation between two correlators involving two or three planes, which each have strong signal. For example, the first pair of four-plane correlators

$$
\begin{aligned}
& 2 \Phi_{2}^{*}-3 \Phi_{3}^{*}-4 \Phi_{4}^{*}+5 \Phi_{5}^{*} \\
& \quad=4\left(\Phi_{2}^{*}-\Phi_{4}^{*}\right)-\left(2 \Phi_{2}^{*}+3 \Phi_{3}^{*}-5 \Phi_{5}^{*}\right), \\
& 6 \Phi_{2}^{*}+3 \Phi_{3}^{*}-4 \Phi_{4}^{*}-5 \Phi_{5}^{*} \\
& \quad=4\left(\Phi_{2}^{*}-\Phi_{4}^{*}\right)+\left(2 \Phi_{2}^{*}+3 \Phi_{3}^{*}-5 \Phi_{5}^{*}\right),
\end{aligned}
$$

are very strong (the black circle and blue cross symbols), while second pair

$$
\begin{aligned}
& \Phi_{2}^{*}+6 \Phi_{3}^{*}-4 \Phi_{4}^{*}-6 \Phi_{6}^{*} \\
& \quad=4\left(\Phi_{2}^{*}-\Phi_{4}^{*}\right)-6\left(\Phi_{3}^{*}-\Phi_{6}^{*}\right), \\
& 4 \Phi_{2}^{*}-6 \Phi_{3}^{*}-4 \Phi_{4}^{*}+6 \Phi_{6}^{*} \\
& \quad=4\left(\Phi_{2}^{*}-\Phi_{4}^{*}\right)+6\left(\Phi_{3}^{*}-\Phi_{6}^{*}\right),
\end{aligned}
$$

are somewhat smaller (the green diamond and magenta square symbols) since the $6\left(\Phi_{3}^{*}-\Phi_{6}^{*}\right)$ correlator does not source the elliptic shape. Furthermore, since

$$
\begin{aligned}
\langle\sin 4 & \left.\left(\Phi_{2}^{*}-\Phi_{4}^{*}\right) \sin \left(2 \Phi_{2}^{*}+3 \Phi_{3}^{*}-5 \Phi_{5}^{*}\right)\right\rangle \\
= & \left\langle\cos \left(2 \Phi_{2}^{*}-3 \Phi_{3}^{*}-4 \Phi_{4}^{*}+5 \Phi_{5}^{*}\right)\right\rangle \\
& -\left\langle\cos \left(6 \Phi_{2}^{*}+3 \Phi_{3}^{*}-4 \Phi_{4}^{*}-5 \Phi_{5}^{*}\right)\right\rangle,
\end{aligned}
$$


Fig. 3 The centrality dependence of several three-plane correlators (Color figure online)
Fig. 4 The centrality dependence of several four-plane correlators not involving $\Phi_{1}^{*}$ plane (Color figure online)

$$
\begin{aligned}
\langle\sin 4 & \left.\left(\Phi_{2}^{*}-\Phi_{4}^{*}\right) \sin 6\left(\Phi_{3}^{*}-\Phi_{6}^{*}\right)\right\rangle \\
= & \left\langle\cos \left(\Phi_{2}^{*}+6 \Phi_{3}^{*}-4 \Phi_{4}^{*}-6 \Phi_{6}^{*}\right)\right\rangle \\
& \quad-\left\langle\cos \left(4 \Phi_{2}^{*}-6 \Phi_{3}^{*}-4 \Phi_{4}^{*}+6 \Phi_{6}^{*}\right)\right\rangle
\end{aligned}
$$

each pair of correlators also allow us to infer the relative sign of the two composing correlators. For example, the cen-
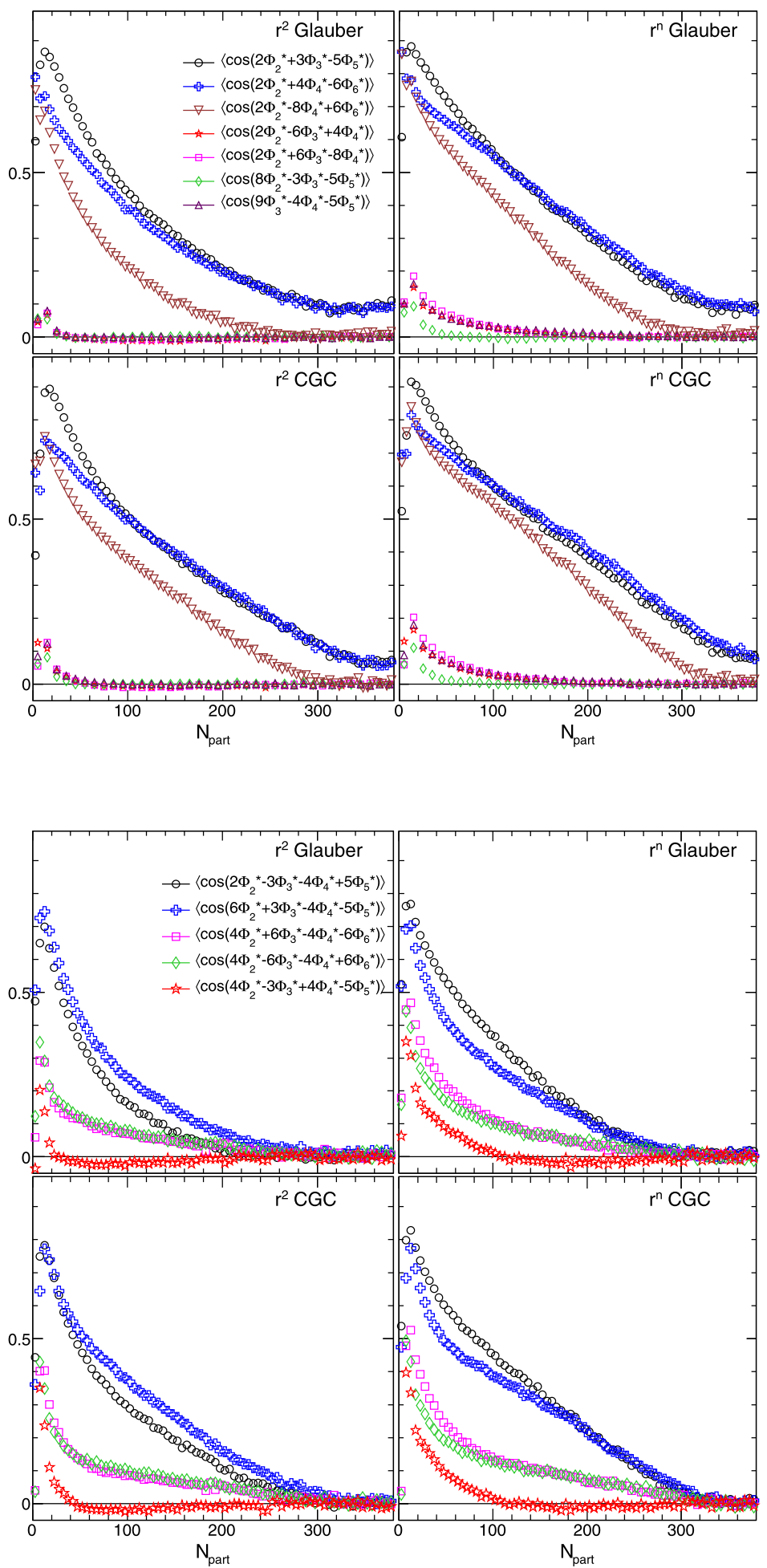

trality dependence of the relative magnitude of both pair of correlators in Fig. 4 suggest that the average values of these sine products are positive in mid-central and peripheral collisions for $r^{n}$-weighting, while they remain negative for $r^{2}$ weighting. The last four-plane correlator (the red star symbols) reflects inter-correlation between two three-plane cor- 
Fig. 5 The centrality dependence of several four-plane correlators involving $\Phi_{1}^{*}$ plane (Color figure online) relators

$$
\begin{aligned}
& 4 \Phi_{2}^{*}-3 \Phi_{3}^{*}+4 \Phi_{4}^{*}-5 \Phi_{5}^{*} \\
& \quad=\left(2 \Phi_{2}^{*}-6 \Phi_{3}^{*}+4 \Phi_{4}^{*}\right)+\left(2 \Phi_{2}^{*}+3 \Phi_{3}^{*}-5 \Phi_{5}^{*}\right),
\end{aligned}
$$

and is small.

Lastly, Fig. 5 shows five four-plane correlators that have a large signal and contain the $\Phi_{1}^{*}$ plane. These curves reflect the correlation between a three-plane correlator and a twoplane correlator

$$
\begin{aligned}
& \Phi_{1}^{*}-2 \Phi_{2}^{*}-3 \Phi_{3}^{*}+4 \Phi_{4}^{*} \\
&=\left(\Phi_{1}^{*}+2 \Phi_{2}^{*}-3 \Phi_{3}^{*}\right)-4\left(\Phi_{2}^{*}-\Phi_{4}^{*}\right), \\
& \Phi_{1}^{*}+6 \Phi_{2}^{*}-3 \Phi_{3}^{*}-4 \Phi_{4}^{*} \\
&=\left(\Phi_{1}^{*}+2 \Phi_{2}^{*}-3 \Phi_{3}^{*}\right)+4\left(\Phi_{2}^{*}-\Phi_{4}^{*}\right), \\
& \Phi_{1}^{*}+2 \Phi_{2}^{*}+3 \Phi_{3}^{*}-6 \Phi_{6}^{*} \\
&=\left(\Phi_{1}^{*}+2 \Phi_{2}^{*}-3 \Phi_{3}^{*}\right)+6\left(\Phi_{3}^{*}-\Phi_{6}^{*}\right), \\
& \Phi_{1}^{*}-2 \Phi_{2}^{*}-4 \Phi_{4}^{*}+5 \Phi_{5}^{*} \\
&=\left(\Phi_{1}^{*}-6 \Phi_{2}^{*}+5 \Phi_{5}^{*}\right)-4\left(\Phi_{2}^{*}-\Phi_{4}^{*}\right), \\
& 3 \Phi_{1}^{*}-2 \Phi_{2}^{*}+3 \Phi_{3}^{*}-4 \Phi_{4}^{*} \\
&=-\left(\Phi_{1}^{*}+2 \Phi_{2}^{*}-3 \Phi_{3}^{*}\right)+4\left(\Phi_{1}^{*}-\Phi_{4}^{*}\right)
\end{aligned}
$$

Since the two composing correlators each have strong signals and are correlated with either the $\Phi_{2}^{*}$-plane (the first four) or the $\Phi_{1}^{*}$ plane (the last one), it is not surprising that these correlators also have sizable signal in mid-central collisions.

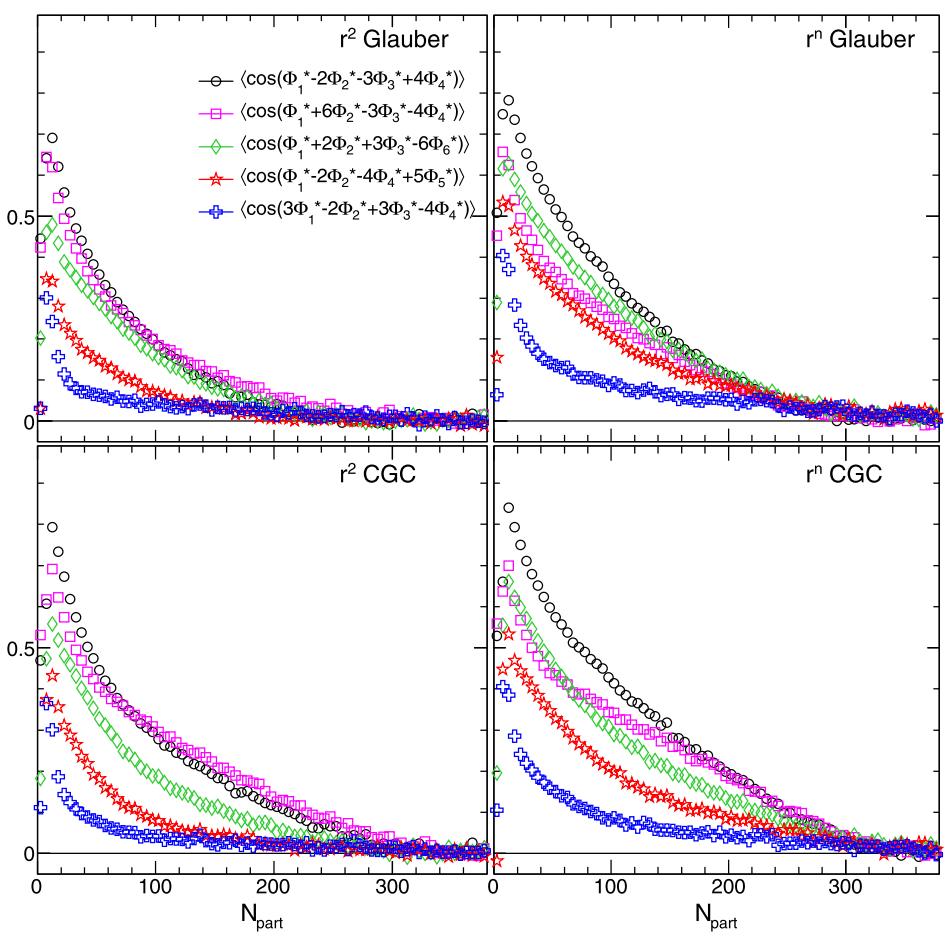

In summary, correlations involving three or four participant planes are investigated in a Glauber model framework. These correlations are calculated in the configuration space, but are expected to contribute to the event plane correlations in momentum space, especially in central collisions. Several significant correlators are identified and the reason for their large magnitudes are clarified. Many of these correlators are expected to have decent resolutions in $\mathrm{Au}+\mathrm{Au}$ or $\mathrm{Pb}+\mathrm{Pb}$ collisions at RHIC and the LHC, so should be measurable if the signal are as big as predicted by the Glauber model.

Acknowledgements This research is supported by NSF under grant number PHY-1305037, PHY-1019387 and DOE under grant number DE-FG02-08ER41540.

Open Access This article is distributed under the terms of the Creative Commons Attribution License which permits any use, distribution, and reproduction in any medium, provided the original author(s) and the source are credited.

\section{References}

1. J. Jia, S. Mohapatra, Eur. Phys. J. C 73, 2510 (2013)

2. Z. Qiu, U.W. Heinz, Phys. Rev. C 84, 024911 (2011)

3. F.G. Gardim, F. Grassi, M. Luzum, J.-Y. Ollitrault, Phys. Rev. C 85, 024908 (2012)

4. D. Teaney, L. Yan, Phys. Rev. C 86, 044908 (2012)

5. P. Staig, E. Shuryak, Phys. Rev. C 84, 034908 (2011)

6. D. Teaney, L. Yan, Phys. Rev. C 83, 064904 (2011)

7. G.-Y. Qin, B. Muller, Phys. Rev. C 85, 061901 (2012)

8. R.S. Bhalerao, M. Luzum, J.Y. Ollitrault, Phys. Rev. C 84, 034910 (2011) 
9. R.S. Bhalerao, M. Luzum, J.Y. Ollitrault, Phys. Rev. C 84, 054901 (2011)

10. A.M. Poskanzer, S.A. Voloshin, Phys. Rev. C 58, 1671 (1998)

11. ATLAS Collaboration, Phys. Rev. C 86, 014907 (2012)

12. M.L. Miller, K. Reygers, S.J. Sanders, P. Steinberg, Annu. Rev. Nucl. Part. Sci. 57, 205 (2007)
13. B. Alver, G. Roland, Phys. Rev. C 81, 054905 (2010) [Erratumibid. C 82, 039903 (2010)]

14. T. Hirano, Y. Nara, Phys. Rev. C 79, 064904 (2009)

15. H.J. Drescher, A. Dumitru, A. Hayashigaki, Y. Nara, Phys. Rev. C 74, 044905 (2006)

16. H.J. Drescher, Y. Nara, Phys. Rev. C 75, 034905 (2007) 